\title{
CLASSIFICATION OF INTELLIGENT AGENT NETWORK TOPOLOGIES AND A NEW TOPOLOGICAL DESCRIPTION LANGUAGE FOR AGENT NETWORKS
}

\author{
Hao Lan Zhang, Clement H.C. Leung and Gitesh K. Raikundalia \\ School of Computer Science and Mathematics, Victoria University
}

haolan@sci.vu.edu.au,Clement.Leung@vu.edu.au,Gitesh.Raikundalia@vu.edu.au

Abstract: Topological theory of intelligent agent networks provides crucial information about the structure of agent distribution over a network. Agent network topologies not only take agent distribution into consideration but also consider agent mobility and intelligence in a network. Current research in the agent network topology area adopts topological theory from the distributed system and computing network fields without considering mobility and intelligence aspects. Moreover, current agent network topology theory is not systematic and relies on graph-based methodology, which is inefficient in describing large-scale agent networks. In this paper, we systematically classify the agent network topologies and propose a new description language called Topological Description Language for Agent networks (TDLA), which incorporates the mobility and intelligence characteristics in an agent network.

Key words: Agent network topology, intelligent agent, topological description language 


\section{INTRODUCTION}

The term Agent network topology is derived from mathematical topological theory and this concept overlaps with topological theory in data communication and distributed systems areas.

Applications of multi-agent systems have been arising in many areas. This situation has led to a set of important research problems concerning how an agent network should be designed to perform efficiently and effectively. Thus, we have to consider designing an appropriate network topology before a network, such as an agent network, is actually constructed. Agent network topology analysis enhances agent communication efficiency of an agent network and provides efficient mobility and intelligence to the network.

Existing topological theory in information technology field has been mainly applied to data communication and distributed systems areas for many years and the theory has made some extraordinary contributions. However, as an emerging discipline, the topological theory in multi-agent systems is still preliminary. Existing topological theory cannot fulfil the needs of agent network because an agent network has its specific characteristics, which include: $i$ ) mobility, ii) intelligence, and $i i i)$ flexibility. The research direction of agent network topology needs to follow these three characteristics. Therefore, this paper classifies the current agent network topologies and analyses the mobility of each topology. We also suggest a new method, called Topological Description Language for Agent network (TDLA), to quantify agent network topologies.

\section{RELATED WORK}

Intelligent agent technology, such as multi-agent application, is often considered as a sub-discipline of Distributed Artificial Intelligence (DAl). Research on topological theory in digital network and distributed systems areas including DAI has been carried out (e.g. [1] [2]).

Much work in distributed system and digital network areas can apply to intelligent agent areas, including topological theory. Nelson Minar [2] classifies distributed system topologies into three general and basic categories, including centralized, decentralized, and hybrid topologies. The topological theory developed in Minar's work is not systematic and lacks comprehensive analysis of topological theory. However, it provides some basic ideas about the classification of simple distributed networks and is helpful to the development of topological theory in multi-agent systems.

After the proposal of Small-world theory and its application to complex networks [3] [4] [5], the development of topological theory soon spreads to complex agent networks [6] [7]. Most of the related work in the field emphasises the application of specific topologies, such as Small-world topology or Scale-free topology, to agent networks. This work does not raise the issue of classification of 
agent networks, which limits the research in gaining comprehensive understanding of agent networks. Thus, this paper clarifies current agent network topological theory.

In our previous research work, we have conducted a performance analysis based on three agent topologies in a newly proposed multi-agent-based architecture [8] called Agent-based Open Connectivity for Decision Support System (AOCD).

\section{AGENT NETWORK TOPOLOGIES CLASSIFICATION}

Agent network topology theory is a crucial area in terms of developing an appropriate agent network infrastructure for a specific organization. We systematically classify agent network topologies into two main categories, which are: simple agent network topology and complex agent network topology.

\subsection{Classification of Simple Agent Network Topologies}

In the real world, a network for an industry organization is complex and specific. However, a complex network can be divided into several simple topologies. For instance, Local Area Network (LAN) theory generally defines four basic topologies [1] [9], which include star topology, bus topology, ring topology, and tree topology. Many organizational LAN applications in the field are basically the combinations of these four basic topologies. Based on the traditional topological theory of LAN, Minar [2] suggests four basic simple topologies and two hybrid topologies in evaluating distributed systems topologies. In the multiagent area, we classify the simple agent network topologies into the following categories.

$>$ Centralized agent network topology:
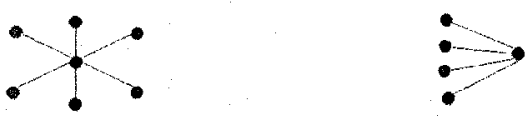

Figure 1. Centralized Topology

Our definition of centralized agent network topology is: a topology has a central agent and only this central agent is connected with other agents over the network. There is no direct connection between any two agents except with the central agent, as shown in Figure 1. A star-like topology is one of the common cases of centralized topology. The total connections in simple centralized topology is:

$$
c=v-1
$$

where $c$ denotes the total connections and $v$ denotes as the total number of agent over a network including the central agent.

In centralized agent network topology, the central agent is vital to the network. However, the central agent has very inefficient mobility. Total connection of a topology is one of the coefficient facts of agent network mobility. We have the 
following equation to define the connection-based coefficient of agent network mobility:

$$
m_{t, 0}=\frac{1}{\sum_{i}(\text { (otalconme ction / import }) \times m_{i}}
$$

where $m_{c o}$ is the connection-degree coefficient of mobility, $m_{i}$ denotes the mobility of an individual agent, import denotes the importance of an agent that is measured by the number of connections to the agent. These variables will be used in the following topologies in this section. Therefore, the connection-based coefficient of centralized agent network mobility is:

$$
m_{a t}=\frac{1}{\sum_{i=1}^{p}\left((v-1) / a_{i}\right) \times m}
$$

where $a_{j}$ is the number of connections for an individual agent. The variables $c, v$, and $a_{i}$ will also be used in the following topologies in this section.

\section{$>$ Peer-to-peer agent network topology:}

There are two categories in Peer-to-peer agent network topology: one is fully connected peer-to-peer topology and another is partially connected peer-to-peer topology.

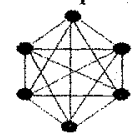

(a) Fully connected

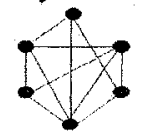

(b) Partially connected

Figure 2. Peer-to-peer agent network topology

In Peer-to-peer agent network topology, each agent has direct connection(s) with other node(s) over a network, as shown in Figure 2. In fully connected peer-to-peer topology, each agent has connections with all the other agents over a network. In partially connected peer-to-peer topology, each agent has at least one connection with another agent over a network and the maximum connections is smaller than $c$. In fully connected peer-to-peer topology, if $v$ denotes the number of agents over a network, then $c$ is given by exhausting all combinations of choosing any two agents:

$$
c=\left(\begin{array}{l}
\prime \prime \\
2
\end{array}\right)=\frac{v(v-1)}{2} \quad 1 \leq v<\infty
$$

The connection-based coefficient of fully connected peer-to-peer agent network mobility is:

$$
m_{c o}=\frac{1}{\sum_{i=1}^{v}\left(v(v-1) / 2 a_{i}\right) \times m_{i}}
$$

\section{$>$ Broadcasting agent network topology:}

We define the broadcasting agent network topology as follows: all the agents are connected through a common media and there is no direct connection between any of the two agents, as shown in Figure 3. Bus topology is a typical broadcasting agent network topology.
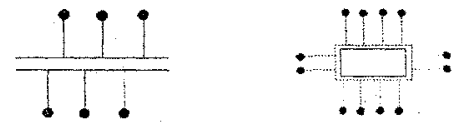

Figure 3. Broadcasting topology 
In broadcasting agent network topology, every agent has same role in the network (unlike in centralized agent network topology, the central agent has a more important role than other agents) and there is no direct connection between any of two agents. The total connections in broadcasting topology is:

$$
c=v
$$

The connection-based coefficient of broadcasting agent network mobility is:

$$
m_{c t r}=\frac{1}{\sum_{i=1}^{v}\left(v / a_{i}\right) \times m_{i}}
$$

\section{Closed-loop agent network topology:}

In closed-loop agent network topology, a network forms a loop and each agent connects exactly to two other agents. In the case of removing connections between any two agents, a closed-loop topology will turn into a linear topology.

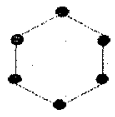

(a) Simple Ring

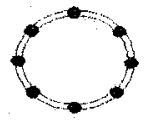

(b) Dual Ring

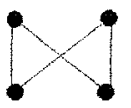

(c) Anomalous closed loop

Figure 4. Closed-Loop topology

As shown in Figure 4 (a) and (b), simple ring and dual ring topologies are the typical closed-loop topologies. Based on our definition, Figure 4 (c) is also a closedloop topology. The total connections in closed-loop topology is:

$$
c=v
$$

The connection-based coefficient of closed-loop agent network mobility is:

$$
m_{e n}=\frac{1}{\sum_{i=1}^{v}\left(v / a_{i}\right) \times m_{i}}
$$

\section{$>$ Linear agent network topology:}

In linear agent network topology, all agents are distributed in a linear form in sequential order and there is no loop in the network. Each agent is connected to two neighbour agents except the two end agents that are only connected to only one neighbour agent.

(a) Simple Linear

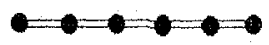

(b) Dual linear

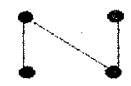

(c) Anomalous linear

Figure 5, Linear topology

In many cases, this topology is regarded as inefficient because the communication between two end agents is extremely inefficient. However, in some cases it appears to be efficient such as in a pipelining process.

The total connections in linear topology is:

$$
c=v-1
$$

The connection-based coefficient of linear agent network mobility is:

$$
m_{v, p}=\frac{1}{\sum_{i=1}^{v}\left((v-1) / a_{i}\right) \times m_{i}}
$$




\section{$>$ Hierarchical agent network topology:}

In hierarchical agent network topology, an agent is the basic unit and a number of agents form a group, which is connected to an upper level agent. In hierarchical agent network topology, an agent is not connected to other agents except to its upper level agent.

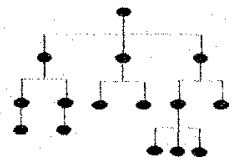

Figure 6. Hierarchical topology

A recursive method can be used to determine if a topology is a hierarchical topology by starting from the end points; which has no lower level agent connected. We define a very important characteristic of hierarchical topology: there is no loop in a hierarchical topology. The total connections in hierarchical topology is:

$$
c=v-1
$$

The connection-based coefficient of hierarchical agent network mobility is:

$$
m_{c o}=\frac{1}{\sum_{i=1}^{v}\left((v-1) / a_{i}\right) \times m_{i}}
$$

Hybrid agent network topology is an important concept in traditional topological theory, which combines two or more simple agent network topologies. Technically speaking, a hybrid agent network topology is still a simple agent network topology compared to complex network topologies from a large-scale point of view. Therefore, a hybrid agent network topology still belongs to the simple agent network topology category.

A hybrid agent network cannot describe an overall complex network in a specific and efficient way, but it explains a complex network in a simple way and it is efficient to a limited scale. A hybrid agent network topology can be a combination of any two or more simple agent network topologies such as a closed loop topology and a simple centralized topology, etc. Hybrid network topology eliminates the difficulties of concurrent control, which mainly plagues centralised topology. Our study shows that hybrid topology offers superior performance in agent-based systems [8]. Nevertheless, simple agent network topological theory (including hybrid topology) can only describe a limited scale of agent networks in a simple way. Hence, current topological theory in the multi-agent field adopts more complex topological theory such as Small-world topology and Scale-free topology to describe a large-scale complex network.

\subsection{Classification of Complex Agent Network Topologies}

Traditional topological theory is insufficient to describe a complex network such as a multi-agent system. This is because current complex networks emphasise the relationships between nodes and traditional topological theory is unable to define the relationships between nodes and describe an overall view of the network efficiently. 
In these circumstances, the topological theory for a complex network is required to provide more abstract descriptions. Current topological theory classifies networks into four major categories:

\section{$>$ Regular Network Topology}

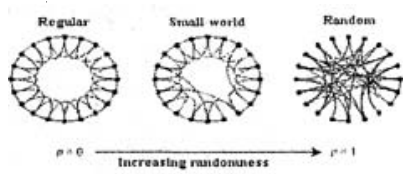

Figure 7. Overview of three complex network topologies (Watt and Strogatz, 1998)

In a regular network, nodes (agents) are distributed in order and the connections between nodes are based on certain constraints. For example, the wiring process is based on finding neighbour agents within the shortest distance. Figure 7 shows an overview of regular network, small-world network and random network [3]. We will explain the transformation process shown on this Figure in the small-world section.

Regular network topology can describe simple networks but it is incapable of describing complex networks efficiently. Generally, regular network topology is limited to describe static networks.

\section{$>$ Random Network Topology}

Random network topology is based on random graph theory, which is described by Paul Erdós and Alfréd Rényi in 1959 [10]. In such a network, the connections between two nodes (agents) are generated randomly.

Considering a graph with vertices contained in a set $X$, as a binary relation $R \subset X \times X$ by defining $R$ as: $(a, b) \in R$ if there is an edge between $a$ and $b$. On the contrary, each symmetric relation $R$ on $X \times X$ gives rise to a graph on $X$. A random graph is a graph $\mathrm{R}$ on an infinite set $X$ satisfying the following properties [11]: i) $\mathrm{R}$ is irreflexive. ii) $\mathrm{R}$ is symmetric. iii) Given any $n+m$ elements $a_{1}, \ldots, a_{n}, b_{1}, \ldots, b_{m} \in X$ there is $c \in X$ such that $c \in X$ is related to $a_{1}, \ldots, a_{n}$, and $c$ is not related to $b_{1}, \ldots, b_{m}$.

Based on random graph theory, a random network topology can describe a largescale complex network. It is more realistic than regular network in describing the real-world complex networks such as multi-agent systems. However, the limitation of random network topology theory is the difficulty of predicting, monitoring and controlling a network. For most agent-based systems this is unacceptable because most of the implemented agent-based systems require a high degree of monitoring and controlling. Therefore, Small-world network topology is suggested.

\section{$>$ Small-World Network Topology}

As we introduced in the previous sections, a regular network topology is easier to monitor and control but it is inefficient in describing a real-world complex network. Oppositely, random network topology has a high degree of disorder, which 
increases the difficulties of operations over the network such as agent search, agent matching, etc.

In the real world, the connection topology is treated as either completely regular or completely random. It is somewhere between these two extreme cases and it is defined by Watts and Strogatz as the Small-world topology [3]. As shown in Figure 7, $p$ denotes the probability of randomness when an agent is connecting with other agents. When $p$ is 0 , a network is wired completely in order. When $p$ is 1 , a network is wired completely randomly. For $0<p<1$, a network is in Small-world topology scope.

Small-world network theory enables the possible control or monitoring over the network especially in some critical areas of a network through observing and adjusting the probability $p$.

Small-world concept is becoming important in multi-agent systems, in which agents are often considered as nodes. It is difficult to use simple or regular agent network topology to describe an overall view of a large multi-agent system. Smallworld topology could efficiently describe the conceptual view of a complex agent network. However, Small-world topology still lacks the ability to adapt to a dynamic environment. In other words, Small-world topology is not an ideal solution for the networks that are constantly changing. Therefore, Scale-free topology is suggested.

\section{$>$ Scale-free Network Topology}

The three topologies we discussed in the previous sections are basically static and homogeneous, and peak at an average value and decay. Such networks are called exponential networks [12]. However, recent research in the field of complex networks indicates that a number of large-scale complex networks including the Internet, WWW, and metabolic networks, are scale-free and the vertices over such a network are not homogeneous.

Barabási and Albert [13] suggest a scale-free network topology, in which a network is allowed to change network connections dynamically and the nodes (agents) on the network are inhomogeneous. The generation scheme of a scale-free network can be summarized into two major steps [12]:

1) Growth: Start with a small number $\left(m_{0}\right)$ of nodes; at every time step, a new node is introduced and is connected to $m \leq m_{0}$ existing nodes.

2) Preferential attachment: The probability $p_{i}$ that a new node will be connected to node $i$ (one of the $m$ existing nodes) depends on the degree $k_{i}$ of node $i$. As a result, $n_{i}=k, \sum, k$, where degree $k_{i}$ is the edges number of a node.

Scale-free network topology is considered as the most suitable topology for multiagent based systems taking high-degree mobility of agent network into account. As we know, one of the most important characteristics of an agent network is that it requires high-degree mobility. To support such a high-degree mobility, a network topology needs the ability of adapting to the dynamic environment and that is the advantage of scale-free topology compared with the other three static topologies. 
Similar to simple agent network topologies, complex agent network topologies are based on graph theory, which is inefficient in describing a large-scale network. An analysis of a network topology is often based on the provided network graph and sometimes lacks precise measurements on each agent. Moreover, the existing agent network topologies are incapable of providing much detailed information of each agent and its relationship with other agents on a network, which increases the difficulty of the operations, such as searching or matching, over a network. Thus, we suggest a description language for agent network topology, called Topological Description Language for Agent networks - TDLA.

\subsection{A Topological Description Language for Agent networks (TDLA)}

Topological Description Language for Agent networks consists of three major sections. These three sections are: i) Individual Agent Description (IAD), ii) Main Agent-groups Description (MAD), iii) Overall Agent-network Description (OAD).

In the IAD section, the description emphasises the information about each agent on a network. The content of IAD includes $(i)$ degree of links (the number of links connected to an agent), (ii) extensibility, which indicates whether an agent allows new connections to be attached and how many connections can be attached, (iii) local address, which is essential for grouping agents by location or generating actual geographic map, (iv) attachment probability and (v) routing table, which stores the information of connected agents to the described agent.

As shown in Figure 8, an example of an IAD expression for the circled agent is as follows. The attachment probability indicates the probability of the selected agent to be attached by other agents. The assumptions are: $(a)$ the nominated node is allowed to attach a maximum of 10 agents, and $(b)$ there are 50 vacant attachments are available in the overall network.

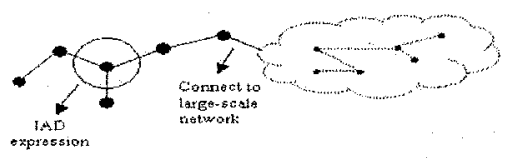

Table 1. IAD Routing Table

Figure 8. IAD for Individual Agent

\begin{tabular}{|c|}
\hline Connected Agent Address \\
\hline 138.77 .201 .38 \\
\hline 138.77 .201 .37 \\
\hline 138.77 .202 .1 \\
\hline
\end{tabular}

(1) Degree of links: 3

(2) Local address: 138.77.201.20

(3) Extensibility: $M C-D L=7$ ( $M C$ : Maximum Connections, $D L$ : Degree of Links)

(4) Attachment probability: Individual Extensibility $/ \sum_{i=1}^{n}(\mathrm{MC}-\mathrm{DL}) \mathrm{i}$

where $n$ is the total number of agents and the individual extensibility is the current agent's extensibility. The result in this case is: $7 / 50=0.14$.

(5) Routing table: See Table 1.

In the MAD section, the description provides network information based on a group of local agents. A large-scale agent network is usually divided into a number 
of sub networks (or groups). The information provided by MAD describes the information of main sub networks. MAD information includes $(i)$ total agent number in a group, (ii) total number of links in a group, (iii) main group selection criteria, (iv) possible root(s) nomination, (v) loop detection in a group and $(v i)$ context within an overall network. The criteria for grouping a number of agents are various, which are based on real cases. The geographic area indication is one of the common criteria for selecting a number of agents as the main agent group. If an agent has much more maximum connection capacity than other agents, this agent normally is nominated as root of the group. In some cases, there is no agent nominated as root. It occurs when the maximum-connection capacity of each agent over a network is equal. To further explain the MAD, we use the previous example and suppose the circled part is a main group of agents as shown in Figure 9.

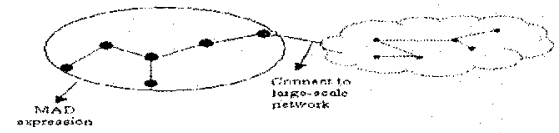

Figure 9. MAD for a main agent-group

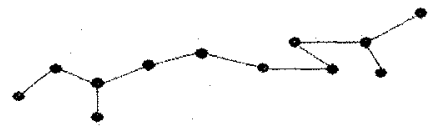

Figure 10. OAD for overall agent network

The MAD is expressed as following.

$\begin{array}{ll}\text { (l) Total number of agents: } 6 & \text { (2) Total number of links in the group: } 6\end{array}$

(3) Main group selection criteria: geography-based in Melbourne/Australia

(4) Root nomination: 138.77 .201 .20 . (5) Loop detection: No loop in the group

(6) Context: main entrance of the state of Victoria.

In the OAD section, the general information of network is provided, which includes (i) the diameter of the network, (ii) total agent number and (iii) info of main agent groups. The diameter of the network is $D=\max _{(i, j)} d(i, j)$, where $i$ and $j$ represent two agents. In other words, the longest path between two agents is the diameter of the network. Using the previous example in Figure 10 to explain the OAD expressions:

$\begin{array}{lll}\text { (I) Diameter of the network: } 9 & \text { (2) Total agent number: } 12\end{array}$

(3) Main agent group info: Total group number: 2

Group 1: (Root nomination: 138.77.201.20)

(Group selection criteria: based in Melbourne)

Group 2: (Root nomination: no root)

(Group selection criteria: geography-based in Brisbane)

Given the descriptions provided by IAD, MAD and OAD, a network generator is able to automatically determine the capability of the network, the preferable area to attach new agents, the topology category (or the combination of topologies) that the network most likely belongs to and the mobility of each agent. In principle, the agent that has a lower degree of connections is likely to have more mobility. TDLA offers the intelligent capability of generating an agent network by using statistical results provided by its three sections. 


\section{CONCLUSION AND FUTURE WORK}

Topological theory in agent networks is an important but somehow underdeveloped research area. In this paper, we classify agent network topologies based on two major categories: simple agent network and complex agent network. In general, we can view a complex agent network as an assemblage of several simple agent networks. However, the difficulties arise when we distinguish the partially connected peer-to-peer network with other simple agent networks. Moreover, existing agent network topologies are graph-based, which are unable to provide detailed information of each agent and its relationship with other agents on a network. Therefore, the Topological Description Language for Agent networks is particularly valuable. The significance of this paper is that it makes a systematic treatment in clarifying and organising the current topological theory in multi-agent field. The proposed TDLA is efficient in constructing agent networks and performing tasks, such as searching and inserting agents in a network. Future work will mainly focus on: (i) experimental design for performance analysis of agent network, and (ii) implementation of TDLA in newly proposed AOCD architecture.

\section{REFERENCES}

1. T. C. Piliouras, "Network Design: Management and Technical Perspective" (2" ed.), pp.141-196. Published by CRC Press LLC, Printed in U.S.A., 2005.

2. N. Minar, "Distributed system topologies," 2002, retrieved on July 2005 from http://www openp2p.com/lpta/1461.

3. D. I. Watts and S.H. Strogatz, "Collective dynamics of 'small world' networks", Nature, vol.393, pp.440-442, June 1998.

4. D.I. Watts, "Small Worlds: The Dynamics of Networks between Order and Randonmess", Princeton University Press, Part 1, 1999.

5. S.H. Strogatz, "Exploring complex networks", Nature, Vol 410, pp.268-276, March 2001.

6. C. Aguirre, J. Martinez-Munoz, F. Corbacho, and R. Huerta, "Small-World Topology for MultiAgent Collaboration", In Proceedings of the $11^{\text {th }}$ International Workshop on Database and Expert Systems Applications, pp. 231-235, 2000.

7. X. Jin and I. Liu, "Agent Network Topology and Complexity", In Proceedings of AAMAS'03, Australia, July 2003.

8. H. L. Zhang, C.H.C. Leung, G. K. Raikundalia, "Performance Analysis of Network Topology in Agent-based Open Connectivity Architecture for DSS", In Proceedings of AINA2006, Vol.2, pp. 257-261, Vienna, Austria, 2006

9. P. Miller and M. Cummins, "LAN Technologies Explained", pp. 6-9. Published by ButterworthHeinemann, Printed in U.S.A. 2000.

10. P. Erdós and A. Rényi, "On the evolution of random graphs", publ. Math. Inst. Hung. Acad. Sci. vol 5, pp. 17-60, 1959.

11. B. Bollobás, "Random Graphs (2"1d Edition)", 2001, Published by Cambridge University Press.

12. X. F. Wang and G. Chen, "Complex Networks: Small-World, Scale-Free and Beyond", IEEE Circuits and systems Magazine, 2003.

13. A-L. Barabási, and R. Albert. "Energence of scaling in random networks", Science, vol. 286, pp. $509-512$, Oct. 1999. 